\title{
Dissipative Optical Breather Molecular Complexes
}

\author{
J. Peng $^{1^{*}}$, Z. Zhao ${ }^{1}$, S. Boscolo ${ }^{2 *}$, C. Finot ${ }^{3}$, S. Sugavanam ${ }^{2}$, D. Churkin ${ }^{4}$, H. Zheng ${ }^{1}$ \\ ${ }^{1}$ State Key Laboratory of Precision Spectroscopy, East China Normal University, Shanghai 200062, China \\ ${ }^{2}$ Aston Institute of Photonic Technologies, Aston University, Birmingham B4 7ET, UK \\ ${ }^{3}$ Laboratoire Interdisciplinaire Carnot de Bourgogne, UMR 6303 CNRS-Université de Bourgogne-Franche-Comté, 21078 Dijon Cedex, France \\ ${ }^{4}$ Novosibirsk State University, Pirogova str. 2, Novosibirsk 630090, Russia \\ jspeng@lps.ecnu.edu.cn; s.a.boscolo@aston.ac.uk
}

\begin{abstract}
We demonstrate different types of breathing soliton complexes in a mode-locked fibre laser: multi-breather molecules, and molecular complexes arising from the binding of two breather-pair molecules or a breather-pair molecule and a single breather. (C) 2020 The Author(s)
\end{abstract}

\section{Introduction}

One of the remarkable properties of dissipative solitons, which are mostly absent in integrable systems, is the ability to form robust multi-pulse bound states. Such soliton complexes, also termed soliton molecules, are currently the subject of intense studies in laser physics by means of real-time ultrafast measurements [1,2], and show dynamics similar to matter molecules, such as synthesis and vibration. While soliton pairs have been the most studied multisoliton structures, a recent study showed that two soliton-pair molecules can bind subsequently to form a stable molecular complex [3]. Apart from parameter-invariant stationary solitons, numerous nonlinear systems support breathing dissipative solitons, manifesting themselves as nonlinear waves in which energy concentrates in a localised and oscillatory fashion [4,5]. Yet, the emergence of bound states of breathing solitons has been less explored than that of their stationary soliton counterparts. A number of recent works have reported on the observation of breathing soliton-pair molecules in mode-locked fibre lasers $[5,6]$.

In the present work, we demonstrate different types of breather complexes in an ultrafast fibre laser: multibreather molecules and molecular complexes originating from the stable binding of two basic molecules, each made up of a pair of breathing solitons $((2+2)$ BMC), or the binding of a breather-pair molecule and a single breather $((2+1) \mathrm{BMC})$. We also explore the non-equilibrium dynamics of BMCs, including collisions of breathers and annihilation of an elementary breather within a BMC. Numerical simulations of the laser model described by the complex Ginzburg Landau equation (CGLE) qualitatively confirm our experimental observations.

\section{Results}

The experimental setup for generating breather complexes is an erbium-doped fibre ring laser, mode-locked by the nonlinear polarisation evolution (NPE) technique and with an anomalous-dispersion cavity. The output signal from the laser is analysed in real time in both the frequency and time domains using dispersive Fourier transform (DFT)based single-shot spectral measurements [7] and spatio-temporal intensity measurements. In our experiment, the pump power is fixed at the value for which the laser operates in the multi-pulse mode-locking regime, and we generate different kinds of breather complexes by tuning the linear cavity loss, hence the pulse interactions, through small rotations of the polarisation controllers (PCs) within the NPE settings.

Figure 1(a-b) shows the dynamics of a breather-quartet molecule ('tetratomic molecule'). The spatio-temporal intensity evolution reveals large periodic variations in the intensities of the four elementary solitons with a period of approximately 1000 round trips. In spite of these intensity variations, the pulse temporal separation remains nearly fixed at approximately $50 \mathrm{ps}$ over consecutive cavity round trips, indicating a strong bond between the pulses. The spectrum features the typical interference pattern that is present in the spectrum of a soliton molecule [1], and the separation between the peaks of spectral intensity matches the pulse spacing in time. The evolution of the spectrum over cavity round trips is periodic, and the spectrum largely widens and narrows within each period, with the broadening (compression) naturally occurring in the vicinity of the position where the pulses reach the highest (lowest) peak intensity. The synchronous oscillation of the central wavelength of the spectrum and the wavelengths of the Kelly sidebands with the pulse energy over cavity round trips [Fig. 1(b)] can be ascribed to cross-phase modulation of the breathers and the radiated dispersive waves [8]. This periodic wavelength shift is mirrored into a periodic shift of the pulse positions in the time domain [Fig. 1(a)], with a positive (to the right) time shift accompanying the pulsations of the individual pulse intensities and no time shift occurring over the duration of the intensity minima.

Another type of a breather complex is observed under different PC settings: a (2+2) BMC consisting of two bound breather pairs. The spatio-temporal intensity dynamics shown in Fig. 1(c) reveal the existence of two characteristic time scales, corresponding to the pulse separation within each breather-pair molecule and the 
separation between the two breather-pair molecules. The intra-molecular pulse separation is nearly the same as that of the breather-quartet molecule, but the inter-molecular separation comes to approximately $350 \mathrm{ps,} \mathrm{which} \mathrm{is} \mathrm{an}$ order of magnitude larger than that of the stationary soliton molecular complexes recently reported [3] and is a signature of long-range interactions. Through rotation of the PCs, we can turn to a different (2+2) BMC [Fig. 1(d)]. Compared to the previous case, the pulses of the two breather-pair molecules are bound at different time intervals, showing that a BMC can have varied intra-molecular separations. By adjusting the cavity loss, we can also decrease the number of breathing solitons and prepare a robust breather triplet. An example is provided in Fig. 1(e), which shows the spatio-temporal evolution of a BMC consisting of a breather-pair molecule bound to a single breather, where the latter can be regarded as a monoatomic molecule. Though the single breather is far apart from the breather pair by about $500 \mathrm{ps,} \mathrm{its} \mathrm{intensity} \mathrm{evolves} \mathrm{synchronously} \mathrm{with} \mathrm{the} \mathrm{breather} \mathrm{pair} \mathrm{over} \mathrm{cavity} \mathrm{round} \mathrm{trips,} \mathrm{indicating} \mathrm{a}$ strong inter-molecular bond. We have also observed a single breather-pair molecule in the laser.
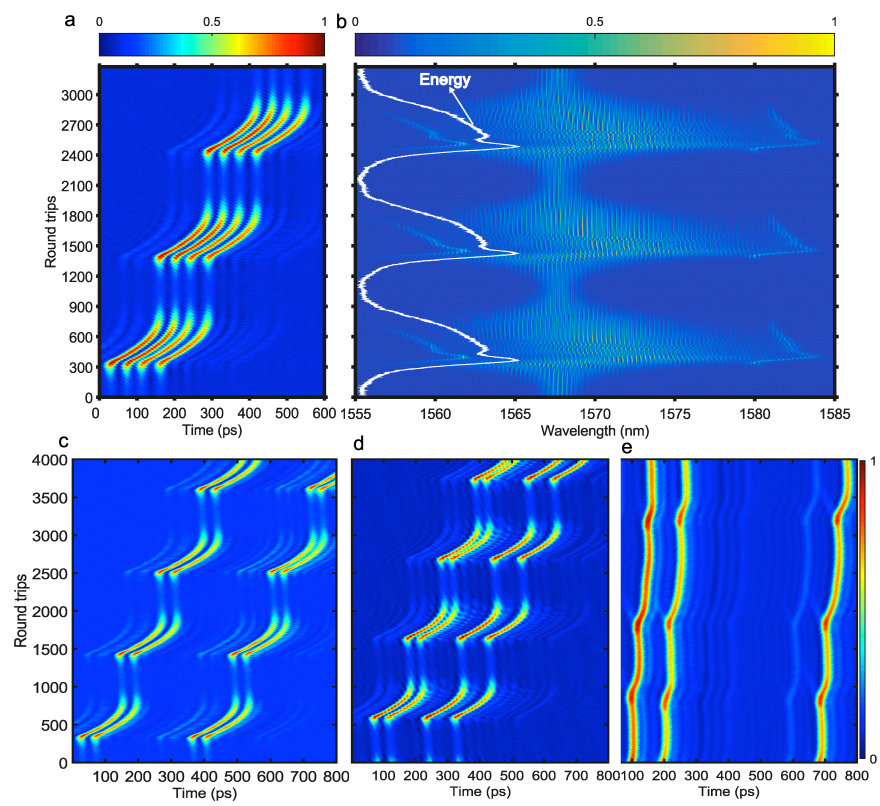

Fig. 1. (a-b) Dynamics of a breathing soliton quartet molecule: (a) Temporal evolution of the intensity relative to the average round-trip time over consecutive round trips; (b) DFT recording of single-shot spectra. The evolution of the energy is also shown (solid white line). (c-e) Dynamics of various breather molecular complexes: Temporal evolution of the intensity relative to the average round-trip time over consecutive round trips for: (c) a $(2+2)$ breather molecular complex with equal intra-molecular pulse separations; (d) a (2+2) breather molecular complex with different intra-molecular separations; and (e) a $(2+1)$ breather molecular complex.

By solving the cubic-quintic CGLE numerically, we have found all the types of breather complexes generated in the experiment. Furthermore, we have observed experimentally breather annihilation within an unstable molecular complex when the complex is in the phase of intensity drop.

\section{Conclusion}

We have pushed the similarity in collective behavior between breathing and stationary dissipative solitons further by reporting on the experimental observation and real-time dynamic characterisation of different types of breather complexes in a passively mode-locked fibre laser. These include tetratomic molecules, and molecular complexes formed from the binding of two diatomic molecules or a diatomic and a monoatomic molecule. We have also observed non-equilibrium dynamics of breathers, including collisions and annihilation. Since breathing dissipative solitons are fundamental modes of many nonlinear physical systems, it is reasonable to assume that the breather dynamics observed in this work will incentivise the investigation of BMCs in various other systems.

\section{References}

[1] G. Herink et al., "Real-time spectral interferometry probes the internal dynamics of femtosecond soliton molecules," Science 356, 50 (2017).

[2] K. Krupa et al., Real-time observation of internal motion within ultrafast dissipative optical soliton molecules," PRL 118, 243901 (2017).

[3] Z. Wang et al., "Optical soliton molecular complexes in a passively mode-locked fibre laser," Nat. Commun. 10, 830 (2019).

[4] E. Lucas et al., "Breathing dissipative solitons in optical microresonators," Nat. Commun. 8, 736 (2017).

[5] J. Peng et al., "Breathing dissipative solitons in mode-locked fiber lasers," Sci. Adv. 5, eaax1110 (2019).

[6] X. Wang et al., "Real-time observation of dissociation dynamics within a pulsating soliton molecule," Opt. Express 27, 28214 (2019).

[7] K. Goda and B. Jalali, "Dispersive Fourier transformation for fast continuous single-shot measurements," Nat. Photon. 7, 102 (2013).

[8] Y. Du et al., "Pulsating soliton with broadened Kelly sidebands in an ultrafast fiber laser," Opt. Lett. 44, 4087 (2019). 